\title{
Statistics of noise-driven coupled nonlinear oscillators: applications to systems with Kerr nonlinearity
}

\author{
Jaroslaw E. Prilepsky ${ }^{a}$ and Stanislav A. Derevyanko b,1 \\ ${ }^{a}$ B.I. Verkin Institute for Low Temperature Physics and Engineering, NASU, 47 \\ Lenin Av., 61103, Kharkov, Ukraine \\ b Photonics Research Group, Aston University, Birmingham, UK, B4 7ET
}

\begin{abstract}
We present exact analytical results for the statistics of nonlinear coupled oscillators under the influence of additive white noise. We suggest a perturbative approach for analysing the statistics of such systems under the action of a determanistic perturbation, based on the exact expressions for probability density functions for noise-driven oscillators. Using our perturbation technique we show that our results can be applied to studying the optical signal propagation in noisy fibres at (nearly) zero dispersion as well as to weakly nonlinear lattice models with additive noise. The approach proposed can account for a wide spectrum of physically meaningful perturbations and is applicable to the case of large noise strength.
\end{abstract}

Key words: Stochastic dynamics, nonlinear oscillators, Fokker-Planck equation, nonlinear optics

PACS: 05.10.Gg, 42.81.Dp

\section{Introduction}

Weakly nonlinear coupled systems (discrete self-trapping model, discrete nonlinear Shrödinger equation, etc.), belong to the universal, widely applicable, highly illustrative and thoroughly studied models of nonlinear physics. These

Email addresses: kovalev@ilt.kharkov.ua (Jaroslaw E. Prilepsky), s.derevyanko@aston.ac.uk (Stanislav A. Derevyanko ).

1 Also at Institute for Radiophysics and Electronics, NAS of Ukraine, 12 Acad. Proscuri St., 61085, Kharkov, Ukraine 
models have applications to molecular crystals, molecular dynamics, nonlinear optics, biomolecular dynamics, and so on (see e.g. [1,2,3,4] and references therein). It was shown that they exhibit unusual dynamical phenomena pertaining only to the nonlinear discrete systems, like existence of intrinsic localised modes etc. [5]. Apart from the dynamical behaviour the influence of random force and statistical properties of nonlinear lattices are also subjects of keen interest $[6,7,8,9]$. The complex dynamics of such systems is determined by an interplay between such factors as randomness, discreetness and nonlinearity. The results concerning the statistics of coupled nonlinear oscillators driven by Gaussian white noise, given below, can be straightforwardly applied to the class of problems which are naturally discrete, e.g. for the noisy self trapping model and its modifications. The second (but not less important) purpose of the current paper is to show that the stochastic discrete models with finite number of degrees of freedom can also be applied to the regularised stochastic continuous systems and can have a much broader field of application. More specifically, in the spirit of the original idea of Mecozzi [10,11], we show that the model of randomly driven coupled nonlinear oscillators can be used for studying the statistics of signal propagation in a nonlinear optical fibre with inline noise sources.

A universal model describing the propagation of the wave envelope in a weakly nonlinear dispersive media (which include optical fibres) is the Nonlinear Shrödinger Equation (NLSE) and its scalar and vector modifications $[12,13,14]$. The need for the profound exploration of noise-stimulated fluctuations of signal parameters in an optical fibre stems from the great practical importance of such study for immediate technical purposes $[12,13]$. The pulse propagation inside an optical fibre with inline amplifiers is described by the perturbed NLSE with additive noise, which accounts for amplifier spontaneous emission (ASE)[12]. It provides an illustrative example of a complicated continuous noisy system where the exact results concerning the statistics of the signal are still very scarce owing to the complexity of the problem (see for instance, review article [15] and also [16,17] for recent results). However if we assume that the noise is delta correlated in both time and space a natural regularisation occurs, which transforms our continuous system into a system of coupled nonlinear oscillators subjected to an external white noise. The statistical properties of such regularised discrete system are much easier to derive than those of the original continuous systems. In particular one can use the Fokker-Planck equation (FPE) approach to obtain the evolution of the probability density function (PDF) for all discrete components of the signal. But even the discrete system corresponding to noisy NLSE and its modifications is still very complicated because of the coupling between the oscillators, which is due to the dispersion. Therefore Mecozzi [10,11] considered propagation of an optical pulse affected by joint action of Kerr nonlinearity and additive white Gaussian noise (WGN) at zero dispersion, i.e. the dispersive (second time derivative) term in the NLSE was dropped. The resulting effective dispersion free equa- 
tion (after the regularisation procedure) formally coincides with the dynamical equation for a single nonlinear oscillator driven by a noisy external force.

In the present paper we treat a zero dispersion stochastic system (a single noise-driven nonlinear oscillator) and its straightforward generalisations (nonlinearly coupled noisy oscillators) as basic systems and consider various small deterministic perturbations which can be of quite arbitrary nature: higher order nonlinearities, small residual dispersion, etc. In particular we can add a perturbation which will be the direct discrete analogue of that of the corresponding continuous optical system. Other continuous models yielding nonlinear discrete equations addressed in the current paper, include higher dimensional systems of nonlinearly coupled NLSEs. Two nonlinearly coupled oscillators with particular coupling coefficients correspond to well known Manakov equations (see e.g. [18]) with zero dispersion driven by white noise. This continuous model describes the nonlinear pulse propagation in a noisy birefringent fibre at nearly zero average dispersion. Systems of more than three coupled NLSEs are also of physical relevance [19]. In addition to optical communications, in the context of biophysics the case of three nonlinearly coupled NLSEs can be applied for studying the propagation of solitons along the three spines of an alpha helix in protein [20]. Systems involving higher numbers of coupled NLSEs have applications in the theory of optical soliton wavelength division multiplexing [21], multichannel biparallel-wavelength optical fibre networks [22], and so on. Hence we believe that the study of the stochastic properties of such models (which, to the best of our knowledge, is a relatively undeveloped field) is also of great interest and may have a significant practical outcome.

As mentioned earlier, we describe the system statistics in terms of the probability density function for the discrete variables and the evolution of the PDF is governed by the FPE [23]. In the case of an unperturbed system of noisedriven nonlinear oscillators (which is a discrete analogue of a system of zero dispersion noisy NLSEs), the corresponding FPE can be solved analytically. The main idea of our approach is to apply the perturbation theory directly to the FPE rather than to the initial stochastic system, using analytical results for the base system. The advantage of such a method is that it allows one to obtain the PDF for the signal output directly as a series in powers of a small parameter $\varepsilon$, which is the effective "strength" of the perturbation. The perturbation theory is based on the propagator of the unperturbed FPE. Once derived, the propagator can be applied to various discrete systems, e.g. regularised systems of weakly coupled NLSE at zero dispersion, where the coupling is considered as a perturbation. This means we can incorporate the effects of coupling and consider the statistics of nonlinear lattice models in the so-called anticontinuum limit (weak intersite linear coupling). In the context of optical applications we are able to consider real polarisation mode dispersion (PMD) systems as well as systems with wavelength division-multiplexing (WDM) [13]. 
The paper is organised in the following way. In section 2 we introduce basic models of one and more noise-driven coupled nonlinear oscillators and write down the Fokker-Planck equation for each model. After that we consider noisy scalar and vector NLSE, describing the propagation of a signal in the optical fibre, and show that after the regularisation (i.e. after the introduction of a discrete time variable) the system becomes equivalent to one of the "base" perturbed systems. We also mention the stochastic discrete self-trapping model as an example of naturally discrete system which can be analysed perturbatively. In section 3 we proceed to find the propagators of FPEs derived for noisy oscillator systems. Using the obtained propagators in section 4 we advance to build a perturbative expansion pf the PDF for different types of perturbations. In this section we consider non-dispersive perturbation of one and more coupled zero dispersion NLSE and provide explicit expressions for several typical example systems. In section 5 we move to more complicated systems, i.e. to the scalar or vector NLSEs, where second dispersion is treated as a perturbation. In the conclusion we summarise the results and outline the key features and perspectives for the approach proposed.

\section{Basic models, equations and regularisation procedure}

In first two subsections we write down the explicit form for the FPEs attached to the system of stochastic equations, governing the evolution of oscillator statistics. Then we explain how these systems can be employed in the context of optical propagation and nonlinear lattices.

\subsection{Single noisy nonlinear oscillator}

By adding a white noise term to the (dimensionless) dynamical equation for a nonlinear Kerr oscillator one gains the Langevin equation for the complex field $u=x+\mathrm{i} y$ :

$$
\frac{\mathrm{d} u}{\mathrm{~d} \zeta}=\mathrm{i}|u|^{2} u+\eta(\zeta)
$$

In the mechanical interpretation $x$ is a position and $y$ is a velocity of the nonlinear oscillator or $x$ and $y$ are the components of a torque for the nonlinear rotator; $\zeta$ has a meaning of time and $\eta(\zeta)=\eta_{1}(\zeta)+\mathrm{i} \eta_{2}(\zeta)$ is the complex white Gaussian noise with the following correlation properties:

$$
<\eta_{i}(\zeta) \eta_{j}\left(\zeta^{\prime}\right)>=2 D \delta_{i j} \delta\left(\zeta-\zeta^{\prime}\right)
$$

with $D$ being the noise strength. Equivalently one can decompose the real and imaginary parts of (1) and obtain a system of two coupled Langevin equations: 


$$
\frac{\mathrm{d} x}{\mathrm{~d} \zeta}=-\left(x^{2}+y^{2}\right) y+\eta_{1}(\zeta), \quad \frac{\mathrm{d} y}{\mathrm{~d} \zeta}=\left(x^{2}+y^{2}\right) x+\eta_{2}(\zeta)
$$

Introducing the $2 \mathrm{D}$ vectors $\boldsymbol{q}=\{x, y\}$ and $\boldsymbol{\eta}=\left\{\eta_{1}, \eta_{2}\right\}$ we can write equation (3) in general vector form:

$$
\frac{\mathrm{d} \boldsymbol{q}}{\mathrm{d} \zeta}=\boldsymbol{f}(\boldsymbol{q})+\boldsymbol{\eta}(\zeta)
$$

Here $\boldsymbol{f}$ is a deterministic "advection vector" (note that sometimes in the literature it is written with a minus sign). This is the canonical form of the Langevin equation with additive WGN. We will be interested in the statistics of the solution of Eq.(4) for a given $\zeta$. Namely, we will seek the conditional PDF of the vector $\boldsymbol{q}, P(\boldsymbol{q}, \zeta)$, provided that at the initial moment $(\zeta=0)$ the $\mathrm{PDF}$ is given. This function (see [23]) obeys the following second order partial differential equation:

$$
\frac{\partial P}{\partial \zeta}=\sum_{i}\left\{-\partial_{i}\left[f_{i}(\boldsymbol{q}) P\right]+D \partial_{i}^{2} P\right\}
$$

which is a FPE attached to system (4) with the additive white noise, given by (2). Also one has to impose the normalisation condition $\int_{\boldsymbol{q}} P \mathrm{~d} \boldsymbol{q}=1$. A propagator is a special solution of (5) with the initial condition $P(\boldsymbol{q}, 0)=$ $\delta\left(\boldsymbol{q}-\boldsymbol{q}^{0}\right)$, i.e. it corresponds to the conditional PDF provided that at the initial moment the system is in a deterministic state $\boldsymbol{q}^{0}$. The explicit form of the FPE for single oscillator system (1) is:

$$
\frac{\partial P}{\partial \zeta}=D\left(\frac{\partial^{2}}{\partial x^{2}}+\frac{\partial^{2}}{\partial y^{2}}\right) P-\left(x^{2}+y^{2}\right)\left(x \frac{\partial}{\partial y}-y \frac{\partial}{\partial x}\right) P .
$$

The boundary conditions for (6) are chosen as follows: the PDF, $P(\boldsymbol{q}, \zeta)$, must have no singularities and should decrease rapidly as $|\boldsymbol{q}| \rightarrow \infty$ to provide the normalisation.

\subsection{System of nonlinearly coupled oscillators}

Next we study the following system of $M$ coupled nonlinear oscillators:

$$
\begin{aligned}
\frac{\mathrm{d} u_{1}}{\mathrm{~d} \zeta} & =\mathrm{i} u_{1} \sum_{i=1}^{M} a_{1 i}\left|u_{i}\right|^{2}+\eta^{1}(\zeta) \\
\vdots & \\
\frac{\mathrm{d} u_{M}}{\mathrm{~d} \zeta} & =\mathrm{i} u_{M} \sum_{i=1}^{M} a_{M i}\left|u_{i}\right|^{2}+\eta^{M}(\zeta) .
\end{aligned}
$$


Here again $u_{i}=x_{i}+\mathrm{i} y_{i}$, are the complex fields, $a_{i j}$ are real constant coefficients and the correlation properties for complex WGNs $\eta^{k}=\eta_{1}^{k}+\mathrm{i} \eta_{2}^{k}$ are (here and further on the subindexes of complex WGNs, "1" and "2", imply the real and imaginary parts correspondingly):

$$
<\eta_{i}^{k}(\zeta) \eta_{j}^{l}\left(\zeta^{\prime}\right)>=2 D_{k} \delta_{k l} \delta_{i j} \delta\left(\zeta-\zeta^{\prime}\right)
$$

(here no summation over the repeated index $k$ implied). To be more general we suppose that WGNs $\eta^{k}(\zeta)$ may have (possibly) different intensities $D_{k}$. Again we can introduce 2 M-dimensional real vectors $\boldsymbol{q}=\left\{x_{1}, \ldots, x_{M}, y_{1}, \ldots y_{M}\right\}$ and $\boldsymbol{\eta}=\left\{\eta_{1}^{1}, \ldots \eta_{1}^{M}, \eta_{2}^{1}, \ldots \eta_{2}^{M}\right\}$, and after singling out real and imaginary parts in (7) arrive at the generic Langevin equation (4). The FPE for this system has the form

$$
\frac{\partial P}{\partial \zeta}=\sum_{i=1}^{M}\left(D_{i} \Delta_{i}-\sum_{j=1}^{M} a_{i j}\left(x_{j}^{2}+y_{j}^{2}\right)\left[x_{i} \frac{\partial}{\partial y_{i}}-y_{i} \frac{\partial}{\partial x_{i}}\right]\right) P .
$$

Here $\Delta_{i}=\partial^{2} / \partial x_{i}^{2}+\partial^{2} / \partial y_{i}^{2}$. We shall call the number $M$ of nonlinearly coupled equations in (7) the dimensionality of the system (the number of independent variables in the FPE (9) is then $2 M$ ).

\subsection{Regularisation procedure for optical pulse propagation: discrete time}

The propagation of a complex light envelope $u(\zeta, t)$ in a noisy optical fibre line with so-called weak dispersion management (see Refs. $[12,24]$ ) is described by the stochastic NLSE:

$$
\frac{\partial u}{\partial \zeta}=-\mathrm{i} \frac{\bar{\beta}}{2} \frac{\partial^{2} u}{\partial t^{2}}+\mathrm{i}|u|^{2} u+\eta(t, \zeta)
$$

(Note that here $\zeta$ plays the role of coordinate in contrast to the mechanical interpretation of oscillators and "virtual" time is $t$.) In equation (10) the stochastic term $\eta(t, \zeta)$ represents a complex WGN. The following correlation properties are assumed:

$$
\begin{aligned}
& <\eta(t, \zeta)>=<\eta(t, \zeta) \eta\left(t^{\prime}, \zeta^{\prime}\right)>=0 \\
& <\eta^{*}(t, \zeta) \eta\left(t^{\prime}, \zeta^{\prime}\right)>=2 G \delta\left(\zeta-\zeta^{\prime}\right) \delta\left(t-t^{\prime}\right)
\end{aligned}
$$

Here $G$ is a normalised noise strength. Equation (10) describes the pathaveraged model of the dispersion managed optical fibre communication system and, it was shown (see [24] and references therein for details) that this model is applicable when the so-called strength of the map, proportional to the local fibre dispersion, to the dispersion oscillation period and to the squared reciprocal pulse width, is small. This strength parameter characterises the effect of the variation of local dispersion. Parameter $\bar{\beta}$ entering Eq.(10) is just 
a path-averaged dispersion coefficient. A zero dispersion NLSE occurs when the path-averaged dispersion coefficient is equal to zero, $\bar{\beta}=0$. The statistics of system (10) at zero dispersion point were first studied in $[10,11]$ (see also [25]). In these Refs. an analytical formula for the conditional PDF of the output signal $u(t, L)$ given the deterministic input signal $u_{0}(t, 0)$ was obtained.

Here we would like to consider the zero-dispersion NLSE assuming also that there is an addition corresponding to a deterministic perturbation:

$$
\frac{\partial u}{\partial \zeta}=\mathrm{i}|u|^{2} u+\eta(t, \zeta)+\varepsilon \hat{N}[u, \ldots] .
$$

Here $\varepsilon \ll 1$ is intended and let $\hat{N}[. .$.$] be any sort of perturbation of the initial$ zero-dispersion NLSE: it may be either a "nonlocal" operator involving the time $\partial / \partial t$ derivatives $\hat{N}\left[u, u_{t}^{\prime}, u_{t t}^{\prime \prime}, \ldots\right]$, or some kind of a "local" operator, $\hat{N}[u]$. Note that complex WGN defined by expression (11) is equivalent to the two independent real WGN, $\eta(t, \zeta)=\eta_{1}(t, \zeta)+\mathrm{i} \eta_{2}(t, \zeta)$, with

$$
\begin{aligned}
<\eta_{1}(t, \zeta) & >=<\eta_{2}(t, \zeta)>=<\eta_{1}(t, \zeta) \eta_{2}\left(t^{\prime}, \zeta^{\prime}\right)>=0 \\
<\eta_{1}(t, \zeta) \eta_{1}\left(t^{\prime}, \zeta^{\prime}\right) & >=<\eta_{2}(t, \zeta) \eta_{2}\left(t^{\prime}, \zeta^{\prime}\right)>=G \delta\left(\zeta-\zeta^{\prime}\right) \delta\left(t-t^{\prime}\right)
\end{aligned}
$$

Because the white noise is delta correlated in both time and space, it has an infinite average power $\left\langle|\eta(t, \zeta)|^{2}>\right.$. This is the consequence of the idealised character of the white noise. In practice, real noise always has small but finite correlation length $r_{c}$ and correlation time $\tau_{c}$. For ASE in optical fibre transmission links the correlation length is in the order of the average amplifier spacing $L_{a}$, and correlation time is inversely proportional to the bandwidth of the noise: $\tau_{c} \sim B^{-1}$. In what follows we will consider our noise still delta correlated in space. However to make the correspondence between discrete and continuous systems explicit and also to get physically meaningful results we move to the context of discrete signals and introduce discrete time variable $t$. We will consider the case where both signal and noise are entirely contained to the time interval of length $T$. Then, to sample the signal effectively, we need a finite number of discrete samples, separated by an interval $\Delta t \sim \tau_{c} \ll T$. If we put $\Delta t=B^{-1}$, the number of samples is $N=B T$. The signal is represented as $N$-dimensional complex vector, $\boldsymbol{u}=\boldsymbol{x}+\mathrm{i} \boldsymbol{y}$, in the sample space:

$$
u(t, \zeta) \Rightarrow \boldsymbol{u}(\zeta), \quad u_{i}(\zeta)=u(i \Delta t, \zeta), \quad i=1, \ldots N
$$

Analogously one can work in the real $2 N$-dimensional space of real and imaginary parts of vector $\boldsymbol{u}: \boldsymbol{q}=\left\{x_{1}, \ldots, x_{N}, y_{1}, \ldots, y_{N}\right\}$. After the regularisation Eq.(12) is equivalent to the system of equations:

$$
\frac{\mathrm{d} u_{i}}{\mathrm{~d} \zeta}=\mathrm{i}\left|u_{i}\right|^{2} u_{i}+\eta^{i}(\zeta)+\varepsilon \hat{N}_{i}[\boldsymbol{u}]
$$

Vector $\boldsymbol{\eta}(\zeta)=\boldsymbol{\eta}_{1}(\zeta)+\mathrm{i} \boldsymbol{\eta}_{2}(\zeta)$ is a regularised white noise with the same prop- 
erties as given by (13) but with regularised temporal delta functions:

$$
<\eta_{1}^{i}(\zeta) \eta_{1}^{j}\left(\zeta^{\prime}\right)>=<\eta_{2}^{i}(\zeta) \eta_{2}^{j}\left(\zeta^{\prime}\right)>=2 D \delta_{i j} \delta\left(\zeta-\zeta^{\prime}\right)
$$

where we set $D \equiv B G$. The operator $\hat{N}_{i}$ is discretised in time as well.

We call the equation, governing the unperturbed stochastic evolution of each separate sampled component, the base system. For all cases considered in this paper, a base system will be either of the form Eq.(1) or Eq.(7); the corresponding FPE for each base system has either a form (6) or a more general look (9), depending on the dimensionality of the base system.

One can see that each sampling component in Eq.(15) obeys the equation governing the noisy dynamics of a single nonlinear oscillator, Eq.(1): hence the dimensionality of the corresponding base system is one. The complete set of equations for all components of the sampled signal has a form of perturbed Eq.(7) with $M=N$ and an identity matrix of $a$-coefficients: $a_{i j}=\delta_{i j}$. As was noted before, we divide the perturbations in equation (15) into two categories: "local", which depend on the value of the signal at the current moment of time only, i.e. $\hat{N}_{i}[\boldsymbol{u}]=\hat{N}_{i}\left[u_{i}\right]$, and "nonlocal" (as, for instance, dispersion, involving second or higher time derivatives of the signal), which comprise the field values at other sampling points.

The same regularisation procedure can be performed with other models relevant to the noisy signal propagation. For example we can consider the propagation in the birefringent fibres where instead of NLSE, under certain conditions, we have a set of Manakov equations [18] perturbed by noise:

$$
\begin{aligned}
& \frac{\partial u}{\partial \zeta}=-\mathrm{i} \frac{\bar{\beta}}{2} \frac{\partial^{2} u}{\partial t^{2}}+\mathrm{i} u\left(|u|^{2}+|v|^{2}\right)+\eta^{1}(\zeta, t), \\
& \frac{\partial v}{\partial \zeta}=-\mathrm{i} \frac{\bar{\beta}}{2} \frac{\partial^{2} u}{\partial t^{2}}+\mathrm{i} v\left(|u|^{2}+|v|^{2}\right)+\eta^{2}(\zeta, t) .
\end{aligned}
$$

Here $u$ and $v$ stand for complex components of the left and right polarised waves and $\eta^{i}(\zeta, t)$ are the complex WGNs. Without noise addition this system describes the processes of the two-dimensional stationary self-focusing and one-dimensional auto-modulation of electromagnetic waves with arbitrary polarisation. Neglecting the dispersion and adding perturbation, after the regularisation one obviously gains:

$$
\begin{aligned}
& \frac{\mathrm{d} u_{i}}{\mathrm{~d} \zeta}=\mathrm{i}\left(\left|u_{i}\right|^{2}+\left|v_{i}\right|^{2}\right) u_{i}+\eta_{i}^{1}(\zeta)+\varepsilon \hat{N}_{i}^{1}[\boldsymbol{u}, \boldsymbol{v}] \\
& \frac{\mathrm{d} v_{i}}{\mathrm{~d} \zeta}=\mathrm{i}\left(\left|u_{i}\right|^{2}+\left|v_{i}\right|^{2}\right) v_{i}+\eta_{i}^{2}(\zeta)+\varepsilon \hat{N}_{i}^{2}[\boldsymbol{u}, \boldsymbol{v}]
\end{aligned}
$$

In this case, for each separate sampled pair $\left[u_{i}, v_{i}\right]$ (if $\varepsilon$ is again set to zero), we arrive at the system of two nonlinearly coupled oscillators. This is just a 
special case of Eq.(7), where the number of dimensions of the base system is two. The whole system for $K=2 N$ sampled components of the signal ( $N$ is, as previously, the number of the sampling time-points) has the form of Eq.(7) as well. However, as the base system has a dimensionality two, here the coupling matrix $a_{i j}$ is no longer an identity matrix but have a $(2 \times 2)$-block diagonal form. The "local" perturbations for such systems are those which satisfy: $\hat{N}_{i}[\boldsymbol{u}, \boldsymbol{v}]=\hat{N}_{i}\left[u_{i}, v_{i}\right]$.

In the same way one could deal with the systems involving higher number $M$ of coupled NLSEs: the number of equations would simply define the dimensionality $M$ of the corresponding base system. The regularised system for the whole set of time-sampled signal components would possess the matrix $a_{i j}$ in a block-diagonal form, where each block would be an $(M \times M)$ square matrix.

\subsection{Nonlinear lattice model}

The dynamics of a weakly nonlinear lattice is described by the set of differentialdifference equations (the discrete self-trapping equation (DSTE), see Refs.[1,2,4]):

$$
\frac{\mathrm{d} u_{i}}{\mathrm{~d} \zeta}=\mathrm{i}\left|u_{i}\right|^{2} u_{i}+\mathrm{i} \varepsilon \sum_{j} m_{i j} u_{j}+\eta^{i}(\zeta),
$$

where we have also added the white noise terms $\eta^{i}(\zeta)$ at each site (with the same statistical properties as in (16)). In Eq.(19) $m_{i j}$ is a coupling matrix and index $j$ ranges over the whole 1D lattice containing $N$ sites. The generalisation for lattices of higher dimensions is straightforward. In physical applications $m_{i j}$ is real and symmetric; in the case of tridiagonal matrix, i.e. for the nearest neighbor coupling, we recover a particular realisation of DSTE - the discrete nonlinear Shrödinger equation [3].

Equation (19) provides an example of a naturally discrete system with Kerr nonlinearity. If we consider the case of vanishing $\varepsilon$, the so-called anticontinuum limit, we arrive at the system of uncoupled oscillators, which is obviously the unperturbed system (15). Here the dimensionality of the base system is one. The perturbation in Eq.(19) provides an example of a nonlocal perturbation, since it involves other lattice sites.

\subsection{The list of the notations}

In this subsection we summarise the notations that will be used throughout the paper. The aim of the proposed notations and indexing scheme is to demonstrate the general ideology and to underline the similarity of all final ex- 
pressions: very different physical systems after the regularisation yield similar formal expansions for the PDF.

Table 1

Table of notations and indexing scheme used in the paper

\begin{tabular}{|l|l|}
\hline \multicolumn{1}{|c|}{ Notation } & \multicolumn{1}{|c|}{ Meaning } \\
\hline $\boldsymbol{u}, \boldsymbol{v}, u_{i}, v_{i}$ & $\begin{array}{l}\text { The sampled complex field variables. The bold symbols } \\
\text { indicate the whole sets } \boldsymbol{u}=\left\{u_{1}, u_{2}, \ldots\right\} \text { etc. The subscript } \\
\text { indicates the sampling time-point number. }\end{array}$ \\
\hline$x_{i}, y_{i}$ and $r_{i}, \phi_{i}$ & $\begin{array}{l}\text { Real and imaginary parts and modulus-phase representa- } \\
\text { tion of the complex field variables. The subscript shows } \\
\text { the sampling number (in some examples the upper index } \\
\text { will be used as well). }\end{array}$ \\
\hline $\boldsymbol{r}, \boldsymbol{\phi}, \boldsymbol{q}, \tilde{\boldsymbol{q}}$ & $\begin{array}{l}\text { Bold symbols denote sets } \boldsymbol{r} \equiv \quad\left\{r_{1}, r_{2}, \ldots\right\}, \boldsymbol{\phi} \equiv \\
\left\{\phi_{1}, \phi_{2}, \ldots\right\} ; \text { where indicated explicitly, the subindex } \\
\text { shows the dimensionality of the set. Vectors } \boldsymbol{q} \text { and } \tilde{\boldsymbol{q}} \text { are } \\
\text { the joint sets: } \boldsymbol{q}_{K} \equiv\left\{x_{1}, x_{2}, \ldots, x_{K}, y_{1}, y_{2}, \ldots, y_{K}\right\}, \text { and } \\
\tilde{\boldsymbol{q}}_{K} \equiv\left\{r_{1}, r_{2}, \ldots, r_{K}, \phi_{1}, \phi_{2}, \ldots, \phi_{K}\right\} . \text { The superscript } 0 " \\
\text { marks the initial deterministic values. The upper index is } \\
\text { also used to distinguish between the different sets. }\end{array}$ \\
\hline$\hat{\mathcal{L}}_{K}$ & $\begin{array}{l}\text { The coordinate part of the FP-operator. The subscript } \\
\text { shows the }(\text { half }) \text { number of independent variables involved. }\end{array}$ \\
\hline $\begin{array}{l}P_{M}(\boldsymbol{q} ; \zeta), P^{K}(\boldsymbol{q} ; \zeta), \\
\mathcal{P}^{K}(\boldsymbol{q} ; \zeta)\end{array}$ & $\begin{array}{l}P_{M}(\boldsymbol{q} ; \zeta), P^{K}(\boldsymbol{q} ; \zeta) \text { are the PDFs for the base system and } \\
\text { for the whole sampled signal. For convenience with a sub- } \\
\text { script we shall explicitly mark the dimensionality of the } \\
\text { underlying base system } M, \text { i.e. for } P_{M} \text { we have } \boldsymbol{q}=\boldsymbol{q} M \\
\text { A superscript will be used to mark the whole PDF, and to } \\
\text { show the total (half }) \text { number of the independent variables } \\
\text { involved. If the dimensionality of the base system is } M, \text { and } \\
\text { the number of sampling points is } N, \text { then } K=M \times N, \\
\boldsymbol{q}=\boldsymbol{q}_{K} \cdot \text { The calligraphic } \mathcal{P}^{K} \text { will mark the PDFs of the } \\
\text { perturbed systems. The tilde above the PDF will mean the } \\
\text { representation in "polar coordinates", }\{\boldsymbol{x}, \boldsymbol{y}\} \rightarrow\{\boldsymbol{r}, \boldsymbol{\phi}\} .\end{array}$ \\
\hline
\end{tabular}


Table of notations and designations used in the paper (cont'd)

\begin{tabular}{|l|l|}
\hline$\hat{N}_{i}[\ldots], \hat{\mathcal{N}}[\ldots]$ & $\begin{array}{l}\hat{N}_{i}[\ldots] \text { and } \hat{\mathcal{N}}[\ldots] \text { are the regularised perturbation op- } \\
\text { erator and its FP-image correspondingly. The explicit } \\
\text { form of the FP-image is derived using the general rules } \\
\text { for the construction of the FPE [23]. }\end{array}$ \\
\hline $\boldsymbol{\eta}=\left\{\eta^{1}, \eta^{2}, \ldots\right\}$ & $\begin{array}{l}\text { Complex regularised WGN. The superscript is used to } \\
\text { distinguish between the different independent WGNs } \\
\text { entering different equations. }\end{array}$ \\
\hline $\mathcal{G}_{M}(\ldots), \mathcal{G}_{K}(\ldots)$ & $\begin{array}{l}\mathcal{G}_{M}(\ldots) \text { is the propagator of the base system FPE with } \\
\text { the coordinate part } \mathcal{L}_{M} \text {. Symbol } \mathcal{G}_{K} \text { will mark the } \\
\text { propagator of the whole FPE of the entire sampled } \\
\text { system. }\end{array}$ \\
\hline
\end{tabular}

\section{Propagator functions of the unperturbed Fokker-Plank equation}

We have just shown that after the regularisation all the models considered in the previous section yield same type of system of Langevin equations, which is merely a perturbed system of coupled nonlinear oscillators (7). Now consider the statistical properties of unperturbed system (7). The FPE attached to system (7) is of course Eq.(9). In this section we will calculate a propagator for Eq.(9) which will allow us to build an effective perturbative expansion for the PDF in the next sections.

First we note that in some examples (like for instance a scalar regularised zero dispersion NLSE (15) with $\varepsilon=0$ ) the coupling matrix $a_{i j}$ is diagonal, and the dimensionality of the base system is one. In that case FPE (9) admits factorised solutions in the form:

$$
P^{K}\left(\boldsymbol{q}_{K} ; \zeta\right)=P\left(x_{1}, \ldots, x_{K}, y_{1}, \ldots y_{K} ; \zeta\right)=\prod_{i=1}^{K} P_{1}\left(x_{i}, y_{i} ; \zeta\right)
$$

where $P_{1}(x, y ; \zeta)$ is a PDF which satisfies unperturbed FPE for a single oscillator (6). The factorised form of Eq.(20) is a natural manifestation of the fact that for diagonal $a_{i j}$ all nonlinear oscillators in system (7) become dynamically uncoupled and, because of (8), statistically uncorrelated. Therefore in order to study the statistics of such a system it is sufficient to study the statistics of a single oscillator (1). Bearing this in mind we initially consider a FPE for a single oscillator (6) and find its propagator, and then utilise the obtained results to find the propagator of generic system (9) by virtue of Eq.(20).

The same is true for the unperturbed systems for which a base system has a 
higher number of dimensions, say $M$. In such a case the coupling matrix $a_{i j}$ is $(M \times M)$-block-diagonal, and the expression for the total PDF has again a factorised form:

$$
P^{K}\left(\boldsymbol{q}_{K} ; \zeta\right)=P\left(x_{1}, \ldots, x_{K}, y_{1}, \ldots y_{K} ; \zeta\right)=\prod_{i=1}^{N} P_{M}\left(\boldsymbol{q}_{M}^{i} ; \zeta\right)
$$

where $P_{M}\left(\boldsymbol{q}_{M}^{i} ; \zeta\right)$ is now a PDF, which satisfies unperturbed FPE for a system of nonlinearly coupled oscillators (9) and $N$ is the number of samples so that $K=N \times M$. The factorisation in Eq.(21) is possible because each of the $N$ block-systems of $M$ nonlinear oscillators is uncoupled from all other remaining block-systems. Now to gain the statistics of this system we are to study the statistics of coupled oscillators, described by FPE (9). After it is done we employ Eq.(21) to find the final answer for the statistics of the entire set of samples.

\subsection{Propagator of the Fokker-Plank operator for a single oscillator}

Let us study the equation for a single oscillator (6). Proceeding to the polar coordinates $x=r \cos \phi, y=r \sin \phi$ we introduce function $\tilde{P}_{1}(r, \phi ; \zeta)=$ $P_{1}(x(r, \phi), y(r, \phi) ; \zeta)$. Note that function $\tilde{P}_{1}$ is not the PDF in polar coordinates since it does not have the Jacobian $r$ included. For the function $\tilde{P}_{1}$ we now obtain the following equation:

$$
\frac{\partial \tilde{P}_{1}}{\partial \zeta}+\hat{\mathcal{L}}_{1} \tilde{P}_{1}=\frac{\partial \tilde{P}_{1}}{\partial \zeta}+\left[-D \Delta^{r}+r^{2} \frac{\partial}{\partial \phi}\right] \tilde{P}_{1}=0
$$

where

$$
\Delta^{r}=\frac{1}{r} \frac{\partial}{\partial r}\left(r \frac{\partial}{\partial r}\right)+\frac{1}{r^{2}} \frac{\partial^{2}}{\partial \phi^{2}}
$$

is the Laplace operator in polar coordinates. We define a propagator, $\mathcal{G}_{1}$, as a special solution of $(22)$ (as a function of $\{\zeta, r, \phi\}$ ), possessing the following properties:

$$
\begin{aligned}
\left.\mathcal{G}_{1}\left(\zeta, \zeta^{\prime} ; \phi, \phi^{\prime} ; r, r^{\prime}\right)\right|_{\zeta=\zeta^{\prime}} & =\delta\left(\phi-\phi^{\prime}\right) \delta\left(r-r^{\prime}\right) / r \\
\mathcal{G}_{1}\left(\zeta, \zeta^{\prime} ; \phi, \phi^{\prime} ; r, r^{\prime}\right) & =0, \quad \zeta<\zeta^{\prime}
\end{aligned}
$$

Note that propagator (23) coincides with the retarded Green function of Eq. (22). Since our system is uniform in $\zeta$ and $\phi$, the propagator depends only on the differences $\Delta \zeta=\zeta-\zeta^{\prime}$ and $\Delta \phi=\phi-\phi^{\prime}: \mathcal{G}_{1}=\mathcal{G}_{1}\left(\Delta \zeta ; \Delta \phi ; r, r^{\prime}\right)$. To find the propagator we first determine eigenfunctions and eigenvalues of the Fokker-Planck operator $\hat{\mathcal{L}}_{1}$ in $(22)$. The right, $\Psi$, and left, $\tilde{\Psi}$, eigenfunctions are calculated in the Appendix A. Using these eigenfunctions (A.6), (A.7), we 
can write the sought propagator as an eigenvalue expansion:

$$
\mathcal{G}_{1}\left(\Delta \zeta ; \Delta \phi ; r, r^{\prime}\right)=\sum_{n, \nu} \Psi_{n \nu}(r, \phi) \mathrm{e}^{-s_{n \nu} \Delta \zeta} \tilde{\Psi}_{n \nu}^{*}\left(r^{\prime}, \phi^{\prime}\right)
$$

Using identity (A.9) the sum over $n$ can be convoluted yielding the following result:

$$
\begin{aligned}
\mathcal{G}_{1}(\Delta \zeta ; \phi & \left.-\phi^{\prime} ; r, r^{\prime}\right)=\frac{1}{2 \pi D} \sum_{\nu=-\infty}^{\infty} \mathrm{e}^{\mathrm{i} \nu\left(\phi-\phi^{\prime}\right)} \frac{\alpha_{\nu}}{\sinh \left(2 \alpha_{\nu} \Delta \zeta\right)} \\
& \times \exp \left[-\frac{\alpha_{\nu}\left(r^{2}+r^{\prime 2}\right)}{2 D} \operatorname{coth}\left(2 \alpha_{\nu} \Delta \zeta\right)\right] \mathrm{I}_{|\nu|}\left(\frac{\alpha_{\nu} r r^{\prime}}{D \sinh \left(2 \alpha_{\nu} \Delta \zeta\right)}\right) .
\end{aligned}
$$

Here $\mathrm{I}_{|\nu|}$ stands for the $\nu$ th order modified Bessel function of the first kind and the quantities $\alpha_{\nu}$ are defined in the Appendix A. Formula (24) is valid for $\Delta \zeta \geq 0$. For $\Delta \zeta<0$ the propagator, $\mathcal{G}_{1}$, must be equal to zero due to (23). Note that the obtained propagator is symmetric with respect to variables $\left(r, r^{\prime}\right)$ and is normalised to unity: $\int_{0}^{\infty} \mathrm{d} r r \int_{0}^{2 \pi} \mathrm{d} \phi \mathcal{G}_{1}\left(\Delta \zeta ; \phi-\phi^{\prime} ; r, r^{\prime}\right)=1$.

Propagator $P_{1}=\mathcal{G}_{1}\left(\zeta-\zeta^{\prime} ; \phi-\phi^{\prime} ; r, r^{\prime}\right)\left(\right.$ with $r(x, y)=\sqrt{x^{2}+y^{2}}, \phi(x, y)=$ $\arctan (y / x)$ and the same for primed variables) represents the solution of equation (6) provided that at the point $\zeta=\zeta^{\prime}$ our variables have deterministic values: $x=x^{\prime}, y=y^{\prime}$. If we want to apply our results to the regularised scalar NLSE with additive white noise, we must use formula (20). Function $P_{1}$ describing the statistics of the unperturbed NLSE at zero dispersion coincides with that obtained in [11] with a different approach (see also Ref. [25]).

\subsection{Propagator for the Fokker-Planck operator for $M$ nonlinearly coupled oscillators}

In the same way, generalising the derivation of PDF for a single oscillator, we can obtain the PDF $\tilde{P}_{M}\left(\boldsymbol{r}, \boldsymbol{\phi}, \boldsymbol{r}_{0}, \boldsymbol{\phi}_{0} ; \zeta\right)$ for system of $M$ nonlinearly coupled oscillators (see Appendix A). FPE (9) in polar coordinates reads as:

$$
\frac{\partial \tilde{P}_{M}}{\partial \zeta}+\hat{\mathcal{L}}_{M} \tilde{P}_{M}=\frac{\partial \tilde{P}_{M}}{\partial \zeta}+\sum_{i}^{M}\left[-D_{i} \Delta_{i}^{r}+\sum_{j}^{M} r_{i}^{2} a_{j i} \frac{\partial}{\partial \phi_{j}}\right] \tilde{P}_{M}=0
$$

where $\Delta_{i}^{r}$ denotes the Laplacian in polar coordinates $\left\{r_{i}, \phi_{i}\right\}$. Similarly to the case of one oscillator, we introduce propagator $\mathcal{G}_{M}\left(\Delta \zeta ; \boldsymbol{\phi}-\boldsymbol{\phi}^{\prime} ; \boldsymbol{r}, \boldsymbol{r}^{\prime}\right)$ for Eq. (25), which is a solution of (25) for $\Delta \zeta>0$ subject to conditions:

$$
\begin{aligned}
\left.\mathcal{G}_{M}\left(\Delta \zeta ; \boldsymbol{\phi}-\boldsymbol{\phi}^{\prime} ; \boldsymbol{r}, \boldsymbol{r}^{\prime}\right)\right|_{\Delta \zeta=0} & =\prod_{i=1}^{M} \delta\left(\phi_{i}-\phi_{i}^{\prime}\right) \delta\left(r_{i}-r_{i}^{\prime}\right) / r_{i} \\
\mathcal{G}_{M}\left(\Delta \zeta ; \boldsymbol{\phi}-\boldsymbol{\phi}^{\prime} ; \boldsymbol{r}, \boldsymbol{r}^{\prime}\right) & =0, \quad \Delta \zeta<0
\end{aligned}
$$


Again the propagator coincides with the retarded Green function for Eq.(25). Using eigenfunctions (A.11) and convolution formula (A.9) we obtain the following expression:

$$
\begin{aligned}
& \mathcal{G}_{M}\left(\Delta \zeta ; \boldsymbol{\phi}-\boldsymbol{\phi}^{\prime} ; \boldsymbol{r}, \boldsymbol{r}^{\prime}\right)=\frac{1}{(2 \pi)^{M}} \sum_{\nu_{1}=-\infty}^{\infty} \ldots \sum_{\nu_{M}=-\infty}^{\infty} \prod_{i=1}^{M} \frac{1}{D_{i}} \mathrm{e}^{\mathrm{i} \nu_{i}\left(\phi_{i}-\phi_{i}^{\prime}\right)} \\
& \times \frac{\alpha_{i}}{\sinh \left(2 \alpha_{i} \Delta \zeta\right)} \exp \left[-\frac{\alpha_{i}\left(r_{i}^{2}+r_{i}^{\prime 2}\right)}{2 D_{i}} \operatorname{coth}\left(2 \alpha_{i} \Delta \zeta\right)\right] \mathrm{I}_{\left|\nu_{i}\right|}\left(\frac{\alpha_{i} r_{i} r_{i}^{\prime}}{D_{i} \sinh \left(2 \alpha_{i} \Delta \zeta\right)}\right)
\end{aligned}
$$

where quantities $\alpha_{i}$ are defined in Appendix A, see Eq.(A.12). Eq.(27) is one of the major results of the current paper: it generalises the results obtained by Mecozzi $[10,11]$ and Turitsin et al. [25] for a single oscillator. As mentioned earlier, if the matrix coefficients $a_{i j}$ are diagonal, then each coefficient $\alpha_{i}$ in (27) depends on $\nu_{i}$ only, and propagator (27) factorises into the product of $M$ identical functions, each having the form of Eq.(24). However, if the coupling matrix $a_{i j}$ is nondiagonal (the case of nonlinearly coupled oscillators), formula (27) cannot be reduced to a trivial product of identical components.

\section{Perturbational approach for zero-dispersion NLSE}

\subsection{General remarks}

We are now in a position to build a general perturbation theory for the perturbed zero-dispersion NLSE and its generalisations. In this section we will consider perturbations $\hat{N}$ which do not contain a dispersive term. Any perturbation operator $\hat{N}$ in Eq.(12) after regularisation yields a Fokker-Plank image $\hat{\mathcal{N}}$ which will appear in the r.h.s. of Eq.(25), and will retain a small perturbation parameter $\varepsilon$. Instead of equation (25) we arrive at the perturbed equation:

$$
\frac{\partial \tilde{\mathcal{P}}_{K}}{\partial \zeta}+\hat{\mathcal{L}}_{K} \tilde{\mathcal{P}}_{K}=\varepsilon \hat{\mathcal{N}} \tilde{\mathcal{P}}_{K}
$$

where $K=M \times N, M$ is the dimensionality of the base system and $N$ is the number of samples. Since the coupling matrix $a_{i j}$ is block-diagonal, we have $\hat{\mathcal{L}}_{K}=\sum_{i=1}^{N} \hat{\mathcal{L}}_{M}^{i}$. When we consider local perturbations to system (12) (or regularised system (15)), the FP-image of the perturbation also decomposes into a sum of independent operators, $\hat{\mathcal{N}}=\sum_{i=1}^{N} \hat{\mathcal{N}}_{i}$, each acting on a specific set of variables $\left\{\boldsymbol{r}^{i}, \boldsymbol{\phi}^{i}\right\}$ belonging to a separate block. Using propagator (27) we arrive at the following expansion (we write only the first corrections to the unperturbed propagator):

$$
\tilde{\mathcal{P}}^{K}\left(\tilde{\boldsymbol{q}} \mid \tilde{\boldsymbol{q}}^{0} ; \zeta\right)=\tilde{P}^{K}\left(\tilde{\boldsymbol{q}} \mid \tilde{\boldsymbol{q}}^{0} ; \zeta\right)+\varepsilon \tilde{P}_{(1)}^{K}\left(\tilde{\boldsymbol{q}} \mid \tilde{\boldsymbol{q}}^{0} ; \zeta\right)+\mathcal{O}\left(\varepsilon^{2}\right)
$$


where for $\tilde{P}_{(1)}^{K}$ we have:

$$
\begin{gathered}
\tilde{P}_{(1)}^{K}\left(\tilde{\boldsymbol{q}} \mid \tilde{\boldsymbol{q}}^{0} ; \zeta\right)=\int_{0}^{\zeta} \mathrm{d} \zeta^{\prime} \int_{Q} \mathrm{~d} \tilde{\boldsymbol{q}}_{K}^{\prime} \mathcal{G}_{K}\left(\zeta-\zeta^{\prime} ; \boldsymbol{\phi}_{K}-\boldsymbol{\phi}_{K}^{\prime} ; \boldsymbol{r}_{K}, \boldsymbol{r}_{K}^{\prime}\right) \\
\times \hat{\mathcal{N}}^{\prime}\left[\mathcal{G}_{K}\left(\zeta^{\prime} ; \boldsymbol{\phi}_{K}^{\prime}-\boldsymbol{\phi}_{K}^{0} ; \boldsymbol{r}_{K}^{0}, \boldsymbol{r}_{K}^{\prime}\right)\right] \\
\mathcal{G}_{K}\left(\zeta-\zeta^{\prime} ; \boldsymbol{\phi}_{K}-\boldsymbol{\phi}_{K}^{\prime} ; \boldsymbol{r}_{K}, \boldsymbol{r}_{K}^{\prime}\right)=\prod_{i=1}^{N} \mathcal{G}_{M}\left(\zeta-\zeta^{\prime} ; \boldsymbol{\phi}_{M}^{i}-\boldsymbol{\phi}_{M}^{i^{\prime}} ; \boldsymbol{r}_{M}^{i}, \boldsymbol{r}_{M}^{i^{\prime}}\right), \\
\int_{Q} \mathrm{~d} \tilde{\boldsymbol{q}}_{K}^{\prime} \equiv \underbrace{\int_{0}^{\infty} \mathrm{d} r_{1}^{\prime} r_{1}^{\prime} \int_{0}^{2 \pi} \mathrm{d} \phi_{1}^{\prime} \ldots \int_{0}^{\infty} \mathrm{d} r_{K}^{\prime} r_{K}^{\prime} \int_{0}^{2 \pi} \mathrm{d} \phi_{K}^{\prime}}_{K}
\end{gathered}
$$

The prime in $\hat{\mathcal{N}}^{\prime}$ indicates that operator $\hat{\mathcal{N}}$ acts on the primed variables. Note that since perturbation enters only advection terms in Langevin equation (4) (or (15)) its Fokker-Planck image has a form of divergence: $\hat{\mathcal{N}}=\vec{\nabla}$. (...) (see equation (5)). Recalling also that the propagator $\mathcal{G}_{M}$ is normalised we can verify from equations (29)-(30) that the overall normalisation of the function $\tilde{\mathcal{P}}_{K}$ is preserved since $\int_{Q} \mathrm{~d} \tilde{\boldsymbol{q}}_{K}^{\prime} \tilde{P}_{(1)}^{K}\left(\tilde{\boldsymbol{q}} \mid \tilde{\boldsymbol{q}}^{\prime} ; \zeta\right)=0$. The PDF in cartesian coordinates $\mathcal{P}^{K}\left(\boldsymbol{q} \mid \boldsymbol{q}^{0} ; \zeta\right)$ can be restored via the substitution

$$
\mathcal{P}^{K}\left(\boldsymbol{q} \mid \boldsymbol{q}^{0} ; \zeta\right)=\tilde{\mathcal{P}}^{K}\left(\boldsymbol{r}(\boldsymbol{x}, \boldsymbol{y}), \boldsymbol{\phi}(\boldsymbol{x}, \boldsymbol{y}) \mid \boldsymbol{r}^{0}\left(\boldsymbol{x}^{0}, \boldsymbol{y}^{0}\right), \boldsymbol{\phi}^{0}\left(\boldsymbol{x}^{0}, \boldsymbol{y}^{0}\right) ; \zeta\right)
$$

In a similar fashion, continuing this recurrent procedure we can find the higher corrections to the PDF (29).

Let us now consider explicitly some examples of nondispersive perturbations.

\subsection{High order nonlinearities}

First we consider the easiest case where the dimensionality of the corresponding base system is one and a perturbation is local. It seems quite natural to choose the higher nonlinear terms as a perturbation for the single zerodispersion noisy NLSE (12):

$$
\hat{N}[u]=\mathrm{i} \sum_{k=1}^{q} c_{k} u|u|^{2 k+2},
$$

where $q$ is any positive integer number and $c_{k}$ are some real phenomenological coefficients. Obviously, the condition $\hat{N}_{i}[\boldsymbol{u}]=\hat{N}_{i}\left[u_{i}\right]$, is fulfilled: this perturbation is local. After the regularisation the FP-image of this operator appears 
as a sum, $\hat{\mathcal{N}}=\sum_{i=1}^{N} \hat{\mathcal{N}}_{i}$, with $N$ being the number of samples and $\hat{\mathcal{N}}_{i}$ given by:

$$
\hat{\mathcal{N}}_{i}=-\sum_{k=1}^{q} c_{k} r_{i}^{2 k+1} \frac{\partial}{\partial \phi_{i}} .
$$

Up to the first order in $\varepsilon$ the solution of (28) is then given by Eq.(29), where one should substitute in Eq.(31) the Green function of the corresponding dimensionality, Eq.(24): $\mathcal{G}_{M} \rightarrow \mathcal{G}_{1}$. Any local perturbation can be handled in the same way.

\subsection{More general systems: two and more zero-dispersion NLSEs coupled via perturbations}

Now we proceed to more complicated systems, e.g. when we need to consider a set of equations (12) with coupling perturbations. In this subsection the number of dimensions of the corresponding base systems is still one, but the perturbation now cannot be treated as local. For instance if we consider two nonlinear couplers or a single birefringent fibre, then in place of Eq.(12) we obtain the following system:

$$
\begin{aligned}
& \frac{\partial u}{\partial \zeta}=\mathrm{i}|u|^{2} u+\eta^{1}(t, \zeta)+\varepsilon \hat{N}^{1}[u(\zeta), v(\zeta)], \\
& \frac{\partial v}{\partial \zeta}=\mathrm{i}|v|^{2} v+\eta^{2}(t, \zeta)+\varepsilon \hat{N}^{2}[u(\zeta), v(\zeta)] .
\end{aligned}
$$

Note that complex noises, $\eta^{1}$ and $\eta^{2}$, are the independent WGNs, and may possibly have different intensities $D_{1}$ and $D_{2}$. The perturbation operators, $\hat{N}^{1}[u, v]$ and (or) $\hat{N}^{2}[u, v]$, are supposed to intermix the components $u$ and $v$ because otherwise the latter would be uncorrelated and one could merely consider the first and the second equations of system (35) independently using the results of the previous subsection.

It is convenient to introduce a second upper index to distinguish the variables for $u$ (superscript "1") and $v$ (superscript "2"), and opt for the following indexing rule: after the regularisation we introduce vectors $\boldsymbol{r}_{2 N}=$ $\left(r_{1}^{1}, \ldots, r_{1}^{N}, r_{2}^{1}, \ldots, r_{2}^{N}\right)$, and $\phi_{2 N}=\left(\phi_{1}^{1}, \ldots, \phi_{1}^{N}, \phi_{2}^{1}, \ldots, \phi_{2}^{N}\right)$, where

$$
\begin{gathered}
r_{1}^{i}=|u(i \Delta t)|, \quad r_{2}^{i}=|v(i \Delta t)|, \quad i=1, \ldots N, \\
\phi_{1}^{i}=\operatorname{Arg}[u(i \Delta t)], \quad \phi_{2}^{i}=\operatorname{Arg}[v(i \Delta t)], \quad i=1, \ldots N .
\end{gathered}
$$

We seek the PDF of perturbed system, Eq.(28): $\tilde{\mathcal{P}}_{K}\left(\boldsymbol{r}_{K}, \boldsymbol{\phi}_{K} \mid \boldsymbol{r}_{K}^{0}, \boldsymbol{\phi}_{K}^{0} ; \zeta\right), K=$ $2 N$. The perturbation in (35) couples the dynamics (and hence the statistics) of variables $\left(r_{1}^{i}, \phi_{1}^{i}\right)$ and $\left(r_{2}^{i}, \phi_{2}^{i}\right)$ for $i=1, \ldots, N$. Since we have only two (possibly) different noise intensities, $D_{1}$ and $D_{2}$, for this case we can rewrite 
general expression (31) in more simple explicit form:

$$
\begin{aligned}
\mathcal{G}_{K}\left(\zeta-\zeta^{\prime} ; \phi_{K}-\phi_{K}^{\prime} ; \boldsymbol{r}_{K}, \boldsymbol{r}_{K}^{\prime}\right)=\prod_{i=1}^{N} \mathcal{G}_{1}^{D_{1}}\left(\zeta-\zeta^{\prime} ; \phi_{1}^{i}-\phi_{1}^{\prime i} ; r_{1}^{i}, r_{1}^{\prime i}\right) \\
\quad \times \mathcal{G}_{1}^{D_{2}}\left(\zeta-\zeta^{\prime} ; \phi_{2}^{i}-\phi_{2}^{\prime i} ; r_{2}^{i}, r_{2}^{\prime i}\right)
\end{aligned}
$$

where the superscripts of the propagators mean that one ought to insert the corresponding noise intensity in the expression for $\mathcal{G}_{1}$, Eq.(24). We can then employ the results of subsection 4.1.

The generalisation for the case when we have $q$ zero-dispersion NLSEs coupled via the $q$-dimensional perturbation operator,

$$
\hat{N}=\left(\hat{N}^{1}, \ldots, \hat{N}^{q}\right)^{T}
$$

is now straightforward. Firstly one should derive the FP-image $\hat{\mathcal{N}}$ for the perturbation $\hat{N}$. Next, by analogy with the case of two equations, one should introduce $K$-dimensional vectors $(K=q \times N): \boldsymbol{r}_{K}=\left(r_{1}, \ldots, r_{K}\right)$ and $\boldsymbol{\phi}_{K}=$ $\left(\phi_{1}, \ldots, \phi_{K}\right)$. Again for the unperturbed system we have $P^{K}\left(\boldsymbol{r}_{K}, \boldsymbol{\phi}_{K} \mid \boldsymbol{q}_{K}^{0}, \boldsymbol{\phi}_{K}^{0} ; \zeta\right)$ in the product form (20). To obtain the expansion for function $\tilde{\mathcal{P}}^{K}$ we then use the formulae given in subsection 4.1. The expression for the propagator can be given in a simplified form similar to Eq.(37): it is a product of $q$ equivalent multipliers $\mathcal{G}_{1}^{D_{k}}, k=1, \ldots, q$, where the corresponding noise intensities $D_{k}$ should be inserted in the expression for the propagator Eq.(24).

\subsubsection{Coupling perturbations of uncoupled systems}

Let us now provide some examples of coupling perturbations.

- For birefringent fibres, with the coupling $(\sim \varepsilon)$ between the different polarisation components of either linearly or circularly polarised light, we have (see e.g. [14]):

$$
\hat{N}[u, v]=\left(\begin{array}{c}
\hat{N}_{1}[u, v] \\
\hat{N}_{2}[u, v]
\end{array}\right)=\mathrm{i}\left(\begin{array}{c}
A u|v|^{2}+B v^{2} u^{*} \\
A v|u|^{2}+B u^{2} v^{*}
\end{array}\right),
$$

where $A$ and $B$ are the (real) constant coefficients of cross-phase modulation and four-waves mixing. The corresponding "polar" FP-image of $\hat{N}$ in Eq. (28) by virtue of indexing scheme (36) can be presented as a sum $\hat{\mathcal{N}}=$ $\sum_{i=1}^{N} \hat{\mathcal{N}}_{i}$, where $N$ is, as usual, the number of time-samples, and for $\hat{\mathcal{N}}_{i}$ we have the following formula: 


$$
\begin{aligned}
\hat{\mathcal{N}}_{i} & =-\left(A+B \cos \left[2\left(\phi_{1}^{i}-\phi_{2}^{i}\right)\right]\right)\left(\left(r_{2}^{i}\right)^{2} \frac{\partial}{\partial \phi_{1}^{i}}+\left(r_{1}^{i}\right)^{2} \frac{\partial}{\partial \phi_{2}^{i}}\right) \\
& +B r_{1}^{i} r_{2}^{i} \sin \left[2\left(\phi_{1}^{i}-\phi_{2}^{i}\right)\right]\left(r_{1}^{i} \frac{\partial}{\partial r_{2}^{i}}-r_{2}^{i} \frac{\partial}{\partial r_{1}^{i}}\right) .
\end{aligned}
$$

- Now consider two nonlinear oscillators with small eigenfrequencies and weak linear coupling (both terms $\sim \varepsilon$ ). This is a special case of self-trapping model [26] in the presence of the additive noise. This system is interesting in itself: it is integrable (in the noiseless case) and possesses a finite degree of freedom analogue of soliton solution (inhomogeneous state). Therefore this system can be reckoned as one of the simplest model examples, where we can investigate the action of noise on an integrable system. The coupling operator $\hat{N}$ now is

$$
\hat{N}[u, v]=\mathrm{i}\left(\begin{array}{c}
-\omega u+\gamma(v-u) \\
-\omega v+\gamma(u-v)
\end{array}\right),
$$

where $\omega$ and $\gamma$ are the real parameters. The number of independent variables is just four: $u \rightarrow\left\{r_{1}, \phi_{1}\right\}, v \rightarrow\left\{r_{2}, \phi_{2}\right\}$. The polar FP-image of $\hat{N}$ takes the form

$$
\begin{aligned}
\hat{\mathcal{N}} & =(\omega+\gamma)\left(\frac{\partial}{\partial \phi_{1}}+\frac{\partial}{\partial \phi_{2}}\right)+\gamma \sin \left(\phi_{2}-\phi_{1}\right)\left(r_{2} \frac{\partial}{\partial r_{1}}-r_{1} \frac{\partial}{\partial r_{2}}\right) \\
& -\gamma \cos \left(\phi_{2}-\phi_{1}\right)\left(\frac{r_{2}}{r_{1}} \frac{\partial}{\partial \phi_{1}}+\frac{r_{1}}{r_{2}} \frac{\partial}{\partial \phi_{2}}\right) .
\end{aligned}
$$

The expression for the propagator is very simple:

$$
\begin{aligned}
\mathcal{G}_{2}\left(\zeta-\zeta^{\prime} ; \phi\right. & \left.-\phi^{\prime} ; \boldsymbol{r}, \boldsymbol{r}^{\prime}\right) \\
& =\mathcal{G}_{1}^{D_{1}}\left(\zeta-\zeta^{\prime} ; \phi_{1}-\phi_{1}^{\prime} ; r_{1}, r_{1}^{\prime}\right) \mathcal{G}_{1}^{D_{2}}\left(\zeta-\zeta^{\prime} ; \phi_{2}-\phi_{2}^{\prime} ; r_{2}, r_{2}^{\prime}\right),
\end{aligned}
$$

where, as before, the expression for each $\mathcal{G}_{1}^{D_{k}}$ is given by Eq.(24).

- Consider a circular nonlinear fibre array comprising $q$ fibres with weak $(\sim \varepsilon)$ linear coupling. Then instead of (35) we acquire a set of $q$ equations for $q$ functions $v_{j}: \boldsymbol{v}=\left(v_{1}, \ldots, v_{q}\right)$. The $q$-dimensional coupling operator now becomes [14]:

$$
\hat{N}[\boldsymbol{v}]=\left(\begin{array}{c}
\hat{N}_{1}[\boldsymbol{v}] \\
\hat{N}_{2}[\boldsymbol{v}] \\
\vdots \\
\hat{N}_{q}[\boldsymbol{v}]
\end{array}\right)=\mathrm{i} C\left(\begin{array}{c}
v_{q}+v_{2} \\
v_{1}+v_{3} \\
\vdots \\
v_{q-1}+v_{1}
\end{array}\right)
$$


with $C$ a real constant. Again it is convenient to introduce the double indexation scheme: the upper index will indicate the sample number, the lower one corresponds to the field number: $v_{1}(i \Delta t) \rightarrow\left\{r_{1}^{i}, \phi_{1}^{i}\right\}, \ldots, v_{q}(i \Delta t) \rightarrow$ $\left\{r_{q}^{i}, \phi_{q}^{i}\right\}$. Then the image of the perturbation (42) can be presented as a sum, $\hat{\mathcal{N}}=\sum_{i=1}^{N} \hat{\mathcal{N}}_{i}$ (with $N$ the number of samples), where

$$
\begin{aligned}
\hat{\mathcal{N}}_{i} & =C \sum_{n=1}^{q}\left[A_{n}^{i}\left(\boldsymbol{r}_{q}^{i}, \phi_{q}^{i}\right)\left(\cos \phi_{n}^{i} \frac{\partial}{\partial r_{n}^{i}}-\frac{\sin \phi_{n}^{i}}{r_{n}^{i}} \frac{\partial}{\partial \phi_{n}^{i}}\right)+B_{n}^{i}\left(\boldsymbol{r}_{q}^{i}, \phi_{q}^{i}\right)\right. \\
& \left.\times\left(\sin \phi_{n}^{i} \frac{\partial}{\partial r_{n}^{i}}+\frac{\cos \phi_{n}^{i}}{r_{n}^{i}} \frac{\partial}{\partial \phi_{n}^{i}}\right)\right]
\end{aligned}
$$

and we used denotations

$$
\begin{aligned}
& A_{n}^{i}\left(\boldsymbol{r}_{q}^{i}, \phi_{q}^{i}\right)=r_{n-1}^{i} \sin \phi_{n-1}^{i}+r_{n+1}^{i} \sin \phi_{n+1}^{i}, \\
& B_{n}\left(\boldsymbol{r}_{q}^{i}, \phi_{q}^{i}\right)=-r_{n-1}^{i} \cos \phi_{n-1}^{i}-r_{n+1}^{i} \cos \phi_{n+1}^{i} .
\end{aligned}
$$

Here we have assumed that $\left(r_{0}^{i}, \phi_{0}^{i}\right) \equiv\left(r_{q}^{i}, \phi_{q}^{i}\right)$ and $\left(r_{q+1}^{i}, \phi_{q+1}^{i}\right) \equiv\left(r_{1}^{i}, \phi_{1}^{i}\right)$. The explicit form for the propagator is now:

$$
\mathcal{G}_{K}\left(\zeta-\zeta^{\prime} ; \phi_{K}-\phi_{K}^{\prime} ; \boldsymbol{r}_{K}, \boldsymbol{r}_{K}^{\prime}\right)=\prod_{i=1}^{N} \prod_{k=1}^{q} \mathcal{G}_{1}^{D_{k}}\left(\zeta-\zeta^{\prime} ; \phi_{k}^{i}-\phi_{k}^{\prime i} ; r_{k}^{i}, r_{k}^{\prime i}\right),
$$

with $K=q \times N$.

\subsubsection{Birefringent fibre with weak four-wave mixing}

So far the original unperturbed system has always been equivalent to the system of uncoupled oscillators, i.e. matrix $a_{i j}$ in (8), (9) and (27) has always been an identity matrix and thus the dimensionality of the base system has been one. This is not the case for a birefringent fibre with a strong cross-

phase modulation, i.e. if the inequality $A \gg \varepsilon$ holds in Eq.(38). Then one has to consider a more general perturbed system at zero dispersion:

$$
\begin{aligned}
& \frac{\partial u}{\partial \zeta}=\mathrm{i}\left(|u|^{2}+A|v|^{2}\right) u+\eta^{1}(t, \zeta)+\varepsilon \hat{N}^{1}[u(\zeta), v(\zeta)], \\
& \frac{\partial v}{\partial \zeta}=\mathrm{i}\left(A|u|^{2}+|v|^{2}\right) v+\eta^{2}(t, \zeta)+\varepsilon \hat{N}^{2}[u(\zeta), v(\zeta)],
\end{aligned}
$$

where $A$ is a real constant. Putting $A=0$ will correspond to system (35). We see that now the dimensionality of the base system is two, the matrix $a_{i j}$ of the base system has the elements $a_{11}=a_{22}=1, a_{12}=a_{21}=A$. As a simplest example of a perturbation for system (44) we choose the four-wave mixing (a special case of (38)):

$$
\hat{N}[u, v]=\mathrm{i} B\left(\begin{array}{c}
v^{2} u^{*} \\
u^{2} v^{*}
\end{array}\right)
$$


with real constant $B$; the intensities of the noises, $\eta^{1}$ and $\eta^{2}$, can, of course, be different: $D_{1}$ and $D_{2}$. Unlike the case of system (38), now the perturbation (45) is a local one: $\hat{N}_{i}[\boldsymbol{u}, \boldsymbol{v}]=\hat{N}_{i}\left[u_{i}, v_{i}\right]$. The "polar" FP-image of $\hat{N}$ has been already derived as a part of expression (39), responsible for the four-wave mixing, i.e. $\hat{\mathcal{N}}=\sum_{i=1}^{N} \hat{\mathcal{N}}_{i}$ with:

$$
\begin{aligned}
\hat{\mathcal{N}} / B & =-\cos \left[2\left(\phi_{1}^{i}-\phi_{2}^{i}\right)\right]\left(\left(r_{2}^{i}\right)^{2} \frac{\partial}{\partial \phi_{1}^{i}}+\left(r_{1}^{i}\right)^{2} \frac{\partial}{\partial \phi_{2}^{i}}\right)+r_{1}^{i} r_{2}^{i} \sin \left[2\left(\phi_{1}^{i}-\phi_{2}^{i}\right)\right] \\
& \times\left(r_{1}^{i} \frac{\partial}{\partial r_{2}^{i}}-r_{2}^{i} \frac{\partial}{\partial r_{1}^{i}}\right) .
\end{aligned}
$$

The propagator is given by

$$
\mathcal{G}_{K}\left(\zeta-\zeta^{\prime} ; \phi_{K}-\phi_{K}^{\prime} ; \boldsymbol{r}_{K}, \boldsymbol{r}_{K}^{\prime}\right)=\prod_{i=1}^{N} \mathcal{G}_{2}\left(\zeta-\zeta^{\prime} ; \phi_{2}^{i}-\boldsymbol{\phi}_{2}^{\prime i} ; \boldsymbol{r}_{2}^{i}, \boldsymbol{r}_{2}^{\prime i}\right)
$$

with the corresponding noise intensities and coupling matrix elements $a_{i j}$ inserted into general definition (27).

The cases of coupling (nonlocal) perturbations and the higher number of dimensions of the corresponding base system can be treated analogously.

\subsection{Discrete self-trapping equation}

It is instructive to give explicit expressions for noisy DSTE. In this system the discreteness is postulated by the model itself, the dimensionality of the base system is one, and we can readily find the perturbation of FP operator applying the results above. Now the perturbation reads (see equation (19)):

$$
\hat{N}[\boldsymbol{u}]=\mathrm{i} \sum_{j=1}^{N} m_{n j} u_{j}
$$

where $N$ defines the number of discrete sites in the chain (lattice). In this case the nonlocal perturbation (48) might couple all $N$ oscillators. Its FP-image is

$$
\hat{\mathcal{N}}=\sum_{n, j=1}^{N} m_{n j} r_{j}\left[\sin \left(\phi_{j}-\phi_{n}\right) \frac{\partial}{\partial r_{n}}-\frac{\cos \left(\phi_{j}-\phi_{n}\right)}{r_{n}} \frac{\partial}{\partial \phi_{n}}\right],
$$

and the propagator of the system factorises into a product of $N$ functions $\mathcal{G}_{1}^{D_{i}}$, with the corresponding noise intensities. We can then apply the general formulae from subsection 4.1 to find the perturbed PDF. 


\section{Second order dispersion as a perturbation}

In this section we will consider more complicated examples of nonlocal perturbations. We will concentrate on one particular type of perturbations which is nevertheless of great practical interest: the dispersive terms in Kerr systems. Such terms can account for, for instance, a small residual dispersion in a dispersion-managed fibre link. To start we first study a scalar case of a single NLSE, and then proceed to vector generalisations, i.e. to the system of coupled NLSEs.

\subsection{Scalar NLSE with weak second order dispersion.}

In this subsection we will consider the perturbation in terms of weak second order dispersion (SOD) in (12), with the dimensionality of the base system being one:

$$
\hat{N}[u]=\mathrm{i} \frac{d}{2} \frac{\partial^{2} u}{\partial t^{2}}
$$

Here $d= \pm 1$ specifies the type of the dispersion (anomalous or normal). For the sake of simplicity we consider the case $d=1$ since the choice of $d=-1$ only alters the sign in the appropriate formulae. As we work with discretely sampled data we need to write down a discrete analogue of the second derivative Eq.(50). To do so we first define the forward finite difference as $\boldsymbol{\delta} f_{n}=f_{n+1}-f_{n}$. The arbitrary precision expansion for the $1 \mathrm{D}$ second derivative, $\partial^{2} u(t) / \partial t^{2}$, reads as [27]:

$$
\left.\frac{\partial^{2} u}{\partial t^{2}}\right|_{t=i \Delta t}=\frac{1}{(\Delta t)^{2}} \ln ^{2}(1+\boldsymbol{\delta}) u_{i}=\frac{1}{(\Delta t)^{2}} \sum_{n=2}^{\infty} b_{n} \boldsymbol{\delta}^{n} u_{i}
$$

where we have applied regularisation procedure (14) and $\boldsymbol{\delta}^{n}$ is the forward difference of the $n$th order:

$$
\boldsymbol{\delta}^{2} u_{i}=u_{i+1}-2 u_{i}+u_{i-1}, \quad \boldsymbol{\delta}^{3} u_{i}=u_{i+2}-3 u_{i+1}+3 u_{i}-u_{i-1}, \quad \text { etc. }
$$

The squared logarithm operator in representation (51) should be treated as a Taylor expansion in powers of $\boldsymbol{\delta}$, and the expansion coefficients $b_{n}$ can be easily calculated: $b_{2}=1, b_{3}=-1, b_{4}=11 / 12$, etc.

Taking into account Eq.(51) we now write the discretised perturbation $\hat{N}_{i}$ as:

$$
(\Delta t)^{2} \hat{N}_{i}[\boldsymbol{u}]=\mathrm{i} \ln ^{2}(1+\boldsymbol{\delta}) u_{i}=\mathrm{i} \sum_{n=2}^{\infty} b_{n} \boldsymbol{\delta}^{n} u_{i}
$$

Further on it is convenient to use the explicit expression for the difference 
operators in terms of binomial decomposition [27]:

$$
\boldsymbol{\delta}^{n} u_{i}=\sum_{j=0}^{n}(-1)^{j}\left(\begin{array}{l}
n \\
j
\end{array}\right) u_{i+n-j}
$$

The Fokker-Planck image of the perturbation $\hat{\mathcal{N}}$ then becomes:

$$
\begin{aligned}
(\Delta t)^{2} \hat{\mathcal{N}} & =\sum_{i=1}^{N} \sum_{n=2}^{\infty} \sum_{j=0}^{n}(-1)^{j}\left(\begin{array}{l}
n \\
j
\end{array}\right) b_{n} r_{i+n-j}\left[\sin \left(\phi_{i+n-j}-\phi_{i}\right) \frac{\partial}{\partial r_{i}}\right. \\
& \left.-\frac{\cos \left(\phi_{i+n-j}-\phi_{i}\right)}{r_{i}} \frac{\partial}{\partial \phi_{i}}\right] .
\end{aligned}
$$

Note that we write an infinite upper limit of summation over the index $n$. However, of course, each difference is limited by the total number of samples $N$, and all the variables outside the sampling interval should be discarded. The form of the perturbative expansion for the full PDF does not differ from that given in previous sections for the non-dispersive perturbations. The total propagator has the form of the $N$-product of functions $\mathcal{G}_{1}$, each having the same noise intensity $D$.

The same procedure may be easily developed if one wishes to account for a perturbative term in the form of a derivative of an arbitrary order with either real or complex coefficient $(\sim \varepsilon)$. For example, the dispersion of arbitrary order $\mathrm{i}^{k} \partial^{k} u / \partial t^{k}$ would result in the change of the power of the logarithm operator in expressions (51),(52). Eventually one should arrive at the same formulae with a different set of coefficients $b_{n}$, which, in that case, have to be the Taylor coefficients for the expansion of the corresponding power of the logarithm operator (one will have to change the lower summation limit for $n$ from 2 to $k$ in Eqs.(51-53) as well). It is worth mentioning that system (15) with the discrete term, given by (52) but truncated at $n=2$, corresponds to the weakly discrete NLSE [3] with additive noise. The increase of the number of terms in expansion (52) (and consequently in (53)) obviously increases the precision at the expense of the computational time.

In the same manner we could redefine the dispersion operator via the backward differences (see [27]) and obtain an expression very similar to (53). If we wish to preserve the time-inversion symmetry, we might use the symmetrised form instead of Eq. $(51): \hat{\mathcal{N}}=\left(\hat{\mathcal{N}}_{\text {forward }}+\hat{\mathcal{N}}_{\text {backward }}\right) / 2$. 


\subsection{Manakov equations with weak second order dispersion}

Next we develop a perturbation theory accounting for the weak dispersion in vector systems, such as Manakov equations (17) (i.e. for the regularised system (18)). Now the number of dimensions of the base system is two and we consider the nonlocal perturbation of the form:

$$
\hat{N}[u, v]=\frac{\mathrm{i}}{2}\left(\begin{array}{c}
\frac{\partial^{2} u}{\partial t^{2}} \\
\frac{\partial^{2} v}{\partial t^{2}}
\end{array}\right) .
$$

We do not present here the explicit expressions for the PDF expansion as they are very similar to those given in the previous subsection, but outline only the general procedure. First we choose the same regularisation we used in subsection 4.3 for two uncoupled equations. Full coupling matrix $a_{i j}$ comprise a set of $2 \times 2$ blocks placed on the main diagonal, with all elements within each block equal to 1 . The expression for the Fokker-Plank image of perturbation (54) is very similar to Eq.(53). The only difference is that now we again have to endow the variables with an additional index to distinguish between the fields: $u_{i} \rightarrow\left(r_{1}^{i}, \phi_{1}^{i}\right), v_{i} \rightarrow\left(r_{2}^{i}, \phi_{2}^{i}\right)$. Now the expression for the FP-image of perturbation (54) consists of the two summands, $\hat{\mathcal{N}}_{1}$ and $\hat{\mathcal{N}}_{2}$, each having the form of Eq.(53): the variables in Eq.(53) for $\hat{\mathcal{N}}_{1}$ should bear additional index "1", and the same applies to $\hat{\mathcal{N}}_{2}$. The propagator for the entire sampled system is a direct product of $\mathcal{G}_{2}^{D_{k}}$, with the corresponding (possibly different) noise intensities, $D_{1}$ and $D_{2}$, and the elements of the coupling matrix for the base system, $a_{11}=a_{12}=a_{21}=a_{22}=1$. All statements from the previous subsection, concerning accounting for either the time inversion symmetry or a higher order dispersion apply to this case as well.

Remark on higher systems of coupled NLSEs. Following the preceding example one can build perturbation expressions for the systems comprising more than two nonlinearly coupled NLSEs. The number of coupled NLSEs, say $M$, simply defines the dimensionality of the base oscillator system. If we have $M$ coupled fields, $v_{1}, \ldots, v_{M}$, then introducing an additional index labelling the field number one finally arrives at the expression for the FP-image of a dispersive perturbation in the form of a sum, $\hat{\mathcal{N}}=\sum_{k} \hat{\mathcal{N}}_{k}$, where each $\hat{\mathcal{N}}_{k}$ has the form of Eq. (53). The propagator for the whole sampled system is again a direct $N$-product of $\mathcal{G}_{M}^{D_{k}}$.

\section{Conclusion}

In the current paper we have proposed an approach for studying the stochastic dynamics of a noise-driven systems with Kerr-type nonlinearity. We presented 
the exact expression for the PDF (and the propagator) for a system of nonlinearly coupled oscillators. Aside from the study of nonlinear lattice models with the additive WGN, we showed how to apply these results to the description of signal propagation in a weakly nonlinear dispersive media driven by WGN. We used the fact that in a system at zero dispersion the PDF for the output signal (field) can be obtained analytically. Using the propagators for a zero dispersion system we have built a perturbation theory and obtained the corrections to the unperturbed PDF in a variety of physically meaningful situations. In particular we were able to obtain the corrections to the PDF in the presence of the nonlocal perturbation, such as a second order dispersion. The knowledge of the output signal statistics allows one to calculate a multitude of important quantities like the probability of error in the fibre optics communication, or, in principle, Shannon capacity of the nonlinear communication channel, modelled by Eq.(12).

Using the discrete time picture one can also examine the statistics of more complex systems than those, described in the current paper, like, for instance, a system of weakly coupled NLSE with (also weak) linear or nonlinear nonlocal perturbations (e.g. nonlinear dispersion). This would simply require increasing the number of dimensions in the corresponding PDF. It is also possible to consider the higher-dimensional noisy NLSE in the same fashion, e.g. the $2+1$ dimensional NLSE where the (discretised) weak second order derivatives are reckoned as a perturbation.

We recognise that for the case of nonlocal perturbations, like higher order dispersion, when the perturbation operator in the FPE becomes quite involved, the corrections to the PDF can only be calculated numerically. Let us recall that the traditional way of calculating the output signal statistics numerically is to use direct Monte Carlo simulations. The latter method, however has a number of significant drawbacks. Normally the calculations are quite time consuming and cannot be used for estimation of the tails of the PDFs, something which is crucial for the performance assessment in the fibre optical communications. Therefore the perturbation technique developed here, with the iteration procedure similar to (30) performed numerically, may present an important method of choice for the systems where the direct Monte Carlo approach fails. The proposed method relies only on the approximate evaluation of integrals and infinite sums without direct integration of the noisy equation. And finally, the pertrubative approach devised in the present paper does not require the noise to be small - which is usually implied in the majority of the approximate methods in stochastic dynamics. Therefore it can be used to analyse systems far from the equilibrium where the impact of noise on the signal degrees of freedom becomes significant.

In conclusion we also point out that the exact analytical results concerning the statistics of nonlinear coupled systems are still few and far between. Thus 
we think that the exact general expression for the propagator of coupled noisy nonlinear oscillators (27) (which coincides with a retarded Green function), is quite important in itself and can serve as a "reference point" for the studies of similar systems.

\section{Acknowledgements}

Authors would like to thank Sergei Turitsyn and Igor Yurkevich for the fruitful discussions and valuable comments. S.D. would like to acknowledge the support from the Leverhulme Trust Project F/00250/B. This work was also supported by NATO Collaborative Linkage Program (No PST.CLG.980068).

\section{A Eigenfunctions of the Fokker-Planck operator}

In this section we calculate the eigenfunctions and eigenvalues of the FPE for single oscillator (22) and for system of nonlinearly coupled oscillators (25). We start from the former case. We wish to solve the eigenvalue problem:

$$
\hat{\mathcal{L}}_{1} \Psi=s \Psi
$$

Since the obvious periodical boundary conditions in $\phi$ it is convenient to perform a Fourier transform with respect to $\phi$ :

$$
\begin{aligned}
\Psi(r, \Delta \phi) & =\sum_{\nu=-\infty}^{\infty} \mathrm{e}^{\mathrm{i} \nu \Delta \phi} \Psi_{\nu}(r) \\
\Psi_{\nu}(r) & =\frac{1}{2 \pi} \int_{0}^{2 \pi} \mathrm{e}^{-\mathrm{i} \nu \Delta \phi} \Psi(r, \Delta \phi) \mathrm{d} \Delta \phi .
\end{aligned}
$$

After the transform the operator $\hat{\mathcal{L}}_{1}$ reads as

$$
\hat{\mathcal{L}}_{1}^{\nu}=-D\left(\frac{1}{r} \frac{\partial}{\partial r}\left(r \frac{\partial}{\partial r}\right)-\frac{\nu^{2}}{r^{2}}\right)+\mathrm{i} \nu r^{2} .
$$

To solve the eigenfunction equation we make the following substitution:

$$
\Psi_{\nu}(y)=y^{|\nu| / 2} \exp \left(-\alpha_{\nu} y / 2\right) \varphi(y)
$$

where $\alpha_{\nu}=(1+\mathrm{i}) \sqrt{\nu D / 2}$ for $\nu>0$ and $\alpha_{\nu}=(1-\mathrm{i}) \sqrt{|\nu| D / 2}$ for $\nu<0$, $y=r^{2} / D$. After all the transformations Eq.(A.1) now has the form:

$$
y \frac{\mathrm{d}^{2} \varphi}{\mathrm{d} y^{2}}+\left(1+|\nu|-\alpha_{\nu} y\right) \frac{\mathrm{d} \varphi}{\mathrm{d} y}+z_{s \nu} \varphi=0,
$$


with $z_{s \nu}=\left(s-2[1+|\nu|] \alpha_{\nu}\right) / 4$. Equation (A.5) has the canonical form of the degenerated hypergeometric equation [28]. Since we want our eigenfunctions to decrease at infinity we must demand that $z_{s \nu} / \alpha_{\nu}=n, n=0,1, \ldots$. This will determine the discrete eigenvalues $s_{n \nu}$ of (A.1), while the corresponding eigenfunctions $\Psi_{n \nu}$ are expressed via generalised Laguerre functions $\mathrm{L}_{\beta}^{\alpha}(\ldots)$ as [28]:

$$
\begin{aligned}
& s_{n \nu}=2 \alpha_{\nu}(2 n+1+|\nu|), \\
& \Psi_{n \nu}(r, \phi)=\mathrm{e}^{\mathrm{i} \nu \phi}\left(\frac{\alpha_{\nu}}{\pi D}\right)^{1 / 2}\left(\frac{n !}{(n+|\nu|) !}\right)^{1 / 2} z^{|\nu| / 2} \exp [-z / 2] \mathrm{L}_{n}^{|\nu|}(z), \\
& z=\alpha_{\nu} r^{2} / D .
\end{aligned}
$$

Operator $\mathcal{L}_{1}$ is of course not Hermitian. Therefore we need to introduce a set of left-eigenfunctions $\tilde{\Psi}_{n \nu}$, which are the eigenfunctions of the adjoint operator $\mathcal{L}_{1}^{\dagger}$ with eigenvalues $\tilde{s}_{n \nu}$ :

$$
\begin{aligned}
& \tilde{s}_{n \nu}=s_{n \nu}^{*}, \\
& \tilde{\Psi}_{n \nu}(r, \phi)=\mathrm{e}^{\mathrm{i} \nu \phi}\left(\frac{\alpha_{\nu}^{*}}{\pi D}\right)^{1 / 2}\left(\frac{n !}{(n+|\nu|) !}\right)^{1 / 2} z^{*|\nu| / 2} \exp \left[-z^{*} / 2\right] \mathrm{L}_{n}^{|\nu|}\left(z^{*}\right) .
\end{aligned}
$$

Eigenfunctions (A.6),(A.7) form a biorthogonal system, i.e.

$$
\int_{0}^{2 \pi} d \phi \int_{0}^{\infty} d r r \tilde{\Psi}_{n \nu}^{*}(r, \phi) \Psi_{n^{\prime} \nu^{\prime}}(r, \phi)=\delta_{n n^{\prime}} \delta_{\nu \nu^{\prime}}
$$

Making use of the identity (see [28]):

$$
\sum_{n=0}^{\infty} n ! \frac{\mathrm{L}_{n}^{\alpha}(u) \mathrm{L}_{n}^{\alpha}(v) w^{n}}{\Gamma(n+\alpha+1)}=\frac{(u v w)^{-\alpha / 2}}{1-w} \exp \left(-w \frac{u+v}{1-w}\right) \mathrm{I}_{\alpha}\left(2 \frac{\sqrt{u v w}}{1-w}\right),|w|<1
$$

analytically extended to the complex plane, in the limit $w \rightarrow 1$ we can obtain the closure relation for the left and right eigenfunctions:

$$
\sum_{n, \nu} \tilde{\Psi}_{n \nu}^{*}(r, \phi) \Psi_{n \nu}\left(r^{\prime}, \phi^{\prime}\right)=r^{-1} \delta\left(r-r^{\prime}\right) \delta\left(\phi-\phi^{\prime}\right)
$$

(factor $r^{-1}$ in the r.h.s. is merely a jacobian).

In the same way, generalising the derivation of the elementary basis (A.6), (A.7), we can built left- and right-eigenfunction for the general $2 M$-dimensional 
operator $\hat{\mathcal{L}}_{M}$ in $(25)$. Using the Dirac notations one gains

$$
\begin{aligned}
\left|n_{1}, \ldots, n_{M}, \nu_{1}, \ldots, \nu_{M}\right\rangle & =\frac{1}{\pi^{M / 2}} \prod_{i=1}^{M} \mathrm{e}^{\mathrm{i} \nu_{i} \phi_{i}} \sqrt{\frac{\alpha_{i}}{D_{i}}}\left[\frac{n_{i} !}{\left(n_{i}+\left|\nu_{i}\right|\right) !}\right]^{1 / 2} \\
& \times z_{i}^{\left|\nu_{i}\right| / 2} \mathrm{e}^{-z_{i} / 2} \mathrm{~L}_{n_{i}}^{\left|\nu_{i}\right|}\left(z_{i}\right) \\
\left\langle n_{1}, \ldots, n_{M}, \nu_{1}, \ldots, \nu_{M}\right| & =\frac{1}{\pi^{M / 2}} \prod_{i=1}^{M} \mathrm{e}^{\mathrm{i} \nu_{i} \phi_{i}} \sqrt{\frac{\alpha_{i}}{D_{i}}}\left[\frac{n_{i} !}{\left(n_{i}+\left|\nu_{i}\right|\right) !}\right]^{1 / 2} \\
& \times z_{i}^{*\left|\nu_{i}\right| / 2} \mathrm{e}^{-z_{i}^{*} / 2} \mathrm{~L}_{n_{i}}^{\left|\nu_{i}\right|}\left(z_{i}^{*}\right) \\
z_{i} & =\alpha_{i} r_{i}^{2} / D .
\end{aligned}
$$

Here the quantities $\alpha_{i}\left(\nu_{1}, \ldots, \nu_{M}\right)$ are defined as:

$$
\alpha_{i}\left(\nu_{1}, \ldots, \nu_{M}\right)=\left\{\begin{array}{c}
(1+\mathrm{i})\left(\frac{D_{i}}{2} \sum_{j=1}^{M} a_{j i} \nu_{j}\right)^{1 / 2} \text { for } \sum_{j=1}^{M} a_{j i} \nu_{j}>0 \\
(1-\mathrm{i})\left(\frac{D_{i}}{2}\left|\sum_{j=1}^{M} a_{j i} \nu_{j}\right|\right)^{1 / 2} \text { for } \sum_{j=1}^{M} a_{j i} \nu_{j}<0
\end{array}\right.
$$

The corresponding (right) eigenvalues of operator $\hat{\mathcal{L}}_{M}$ are

$$
s_{n_{1}, \ldots, n_{M} \nu_{1}, \ldots, \nu_{M}}=2 \sum_{i=1}^{M} \alpha_{i}\left(2 n_{i}+1+\left|\nu_{i}\right|\right) \text {. }
$$

Since we know the explicit expressions for eigenvalues, we know the damping eigenrates $(\gamma=|\operatorname{Re}[s]|)$ for each eigenmode of FPE. These may be important for the investigation of the long scale (large values of $\zeta$ ) evolution of the system statistics, because for the case $\zeta \rightarrow \infty$ the statistics of the system is determined by a single eigenmode with the lowest value of $\gamma$. We can see that the damping rate for each eigenmode is proportional to $\gamma(\nu) \sim D^{1 / 2} \nu^{3 / 2}$ for a single oscillator or, for a particular realisation $\left(\nu_{1}, \ldots, \nu_{N}\right)$, to $\gamma\left(\nu_{1}, \ldots, \nu_{N}\right) \sim$ $\sum_{i, j=1}^{N} D_{i}^{1 / 2} \nu_{i}\left(a_{j i} \nu_{j}\right)^{1 / 2}$ for a system of nonlinearly coupled oscillators. Note that the real parts of eigenvalues (A.6),(A.13) are always positive which means that equations $(22),(25)$ do not possess a stationary solution.

\section{References}

[1] J.C. Eilbeck, Introduction to the discrete self-trapping equation, in P.L. Christiansen and A.C. Scott eds., Davydov's Solitons Revisited (Plenum Press, 1990) 473-483.

[2] J.C. Eilbeck, P.S. Lomdahl, and A.C. Scott, The discrete Self-trapping equation, Physica D 16 (1985) 318-338. 
[3] P.G. Kevrekidis, K. Ø. Rasmussen, and A. R. Bishop, The discrete nonlinear Schrodinger equation: A survey of recent results, Int. J. Mod. Phys. B 15 (2001) 2833-2900.

[4] J.C. Eilbeck and M. Johansson, The Discrete Nonlinear Schrodinger equation 20 Years on, Proc. of the 3rd Conf. Localization and Energy Transfer in Nonlinear Systems, ed L Vázquez et al, World Scientific, New Jersey, 2003, 44-67.

[5] D.K. Campbell, S. Flach and Yu.S. Kivshar, Localizing energy through nonlinearity and discreteness, Phys. Today 57 (2004) 43-49.

[6] K.Ø. Rasmussen, P.L. Christiansen, M. Johansson, Yu.B. Gaididei, and S.F. Mingaleev, Localized excitations in discrete nonlinear Schrodinger systems: Effects of nonlocal dispersive interactions and noise, Physica D 113 (1998) 134-151.

[7] M.I. Molina, and G.P. Tsironis, Absence of localization in a nonlinear random binary alloy, Phys. Rev. Lett. 73 (1994) 464-467.

[8] K.Ø. Rasmussen, T. Cretegny, P.G. Kevrekidis, and N. Gronbeck-Jensen, Statistical mechanics of a discrete nonlinear system, Phys. Rev. Lett. 61 (2000) 3740-3743.

[9] T.D. Frank, Stability analysis of mean feld models described by FokkerPlanck equations, Ann. Phys. (Leipzig) 11 (2002) 707-716.

[10] A.Mecozzi, Long-distance transmission at zero dispersion - combined effect of the Kerr nonlinearity and the noise of the in-line amplifiers, JOSA B 11 (1994) 462-469.

[11] A. Mecozzi, Limits to long-haul coherent transmission set by the Kerr nonlinearity and noise of the in-line amplifierds, J. Lightwave Technol. 12 (1994) 1993-2000.

[12] E. Iannone, F. Matera, A. Mecozzi, and M. Settembre, Nonlinear Optical Communication Networks (John Wiley \& Sons, 1998).

[13] G.P. Agrawal, Nonlinear fiber optics, (Academic Press, San Diego, 2001).

[14] Akhmediev N.N., Ankiewich A, Solitons. Nonlinear pulses and beams, (Chapman \& Hall, 2000).

[15] H.A. Haus, W.S. Wong, Solitons in optical communications, Rev. Mod. Phys. 68 (1996) 423-444.

[16] G. Falkovich, I. Kolokolov, V. Lebedev, V. Mezentsev, and S. Turitsyn, NonGaussian error probability in optical soliton transmission, Physica D 195 (2004) $1-28$.

[17] S.A. Derevyanko, S.K. Turitsyn, and D.A. Yakushev, Non-Gaussian statistics of an optical soliton in the presence of amplified spontaneous emission, Opt. Lett. 28 (2003) 2097 - 2099. 
[18] T.I. Lakoba, D.J. Kaup, Perturbation theory for the Manakov soliton and its applications to pulse propagation in randomly birefringent fibers, Phys. Rev. E 56 (1997) 6147-6165.

[19] T. Kanna and M. Lakshmanan, Exact soliton solutions, shape changing collisions, and partially coherent solitons in coupled nonlinear Schrodinger equations, Phys. Rev. Lett. 86 (2001) 5043-5046.

[20] A. C. Scott, Launching a Davydov soliton 1. Soliton analysis, Phys. Scr. 29 (1984) 279-283.

[21] S. Chakravarty, M. J. Ablowitz, J. R. Sauer, and R. B. Jenkins, Multisoliton interactions and wavelength-division multiplexing, Opt. Lett. 20 (1995) 136-138.

[22] C. Yeh and L. Bergman, Enhanced pulse compression in a nonlinear fiber by a wavelength division multiplexed optical pulse, Phys. Rev. E 57 (1998) 2398-2404.

[23] H. Risken, The Fokker-Planck Equation, (Springer, 1996).

[24] S.K. Turitsyn, E.G. Turitsyna, S.B. Medvedev, and M.P. Fedoruk, Averaged model and integrable limits in nonlinear double-periodic Hamiltonian systems, Phys. Rev. E 61 (2000) 3127-3132.

[25] K.S. Turitsyn, S.A. Derevyanko, I.V. Yurkevich, and S.K. Turitsyn, Information capacity of optical fiber channels with zero average dispersion, Phys. Rev. Lett. 91 (2003) art. no. 203901.

[26] V.M. Kenkre, and D.K. Campbell, Self-trapping on a dimer - time-dependent solutions of a discrete nonlinear Shrodinger equation, Phys. Rev. B. 34 (1986) 4959-4961.

[27] W.F. Ames, Numerical Methods for Partial Differential Equations, (Academic Press, 1977).

[28] I.S. Gradshteyn, I.M. Ryzhik, Alan Jeffrey (Editor), Table of Integrals, Series and Products, (Academic Press, New York, 2002). 